\title{
Reference Object
}

National Cancer Institute

\section{Source}

National Cancer Institute. Reference Object. NCI Thesaurus. Code C48294.

Something referred to; the object of a reference. 